UDC: 515.122 .2

DOI: $10.37560 /$ matbil21452121b

\title{
SOME COVERING PROPERTIES USING MAXIMAL OPEN COVERS
}

\author{
KALLOL BHANDHU BAGCHI ${ }^{1}$
}

\begin{abstract}
The aim of this article is to study some covering properties using maximal open covers in topological spaces. We see that in a $m$-Lindelöf topological space each uncountable subset has a complete $m$-accumulation point as well as $m$-accumulation point. Furthermore we see that a Hausdorff $m$-paracompact topological space is minimal $c$-normal.
\end{abstract}

\section{INTRODUCTION}

We simply write $\mathrm{X}$ to denote a topological space $(X, \mathscr{T})$. By a proper open set (resp., closed set) of a topological space $X$, we mean an open set $G \neq \emptyset, X$ (resp., $E \neq \emptyset, X)$. We also write $|A|$ to denote the cardinality of the subset $A$ of $X$. By $A \subsetneq B$ we mean that $A \subseteq B$ but $A \neq B$.

In recent years, the notions of minimal, maximal and mean open and closed sets become a centre of attraction in the literature. There are significance number of research articles investigating several concepts using minimal, maximal and mean open and closed set. The investigative aspects of such kind of sets are still yielding new and interesting concepts. Nakaoka and Oda [9] introduced and studied the concept of maximal open sets in topological spaces: a proper open set $U$ of a topological space $X$ is said to be a maximal open set if $U$ is contained in an open set $G$ of $X$, then $G=U$ or $G=X$. Therefore if $U$ is a maximal open set in a topological space $X$, there is no proper open set $V$ in $X$ such that $U \subsetneq V$. Considering this nature of maximal open sets, Mukharjee et al. [7] define $s$-refinement (Definition 2.3) which immediately leads to define maximal open covers of topological spaces (Definition 2.4).

\section{BASIC DEFINITIONS AND RESUlTS}

Firstly, we recall the followings.

2010 Mathematics Subject Classification. Primary: 54D10, Secondary: 54D15.

Key words and phrases. $s$-refinement, maximal open cover, $m$-compact space, $m$-Lindelöf space, $m$-paracompact space. 
Definition 2.1 (Nakaoka and Oda, [10]). A proper closed set $E$ of $X$ is said to be a minimal closed set if $F$ is a closed set of $X$ contained in $E$, then $E=\emptyset$ or $E=F$.

Definition 2.2 (Nakaoka and Oda, 8, 9]). A proper open set $U$ of $X$ is said to be a maximal open set if $U$ is contained in an open set $G$ of $X$, then $G=U$ or $G=X$.

Definition 2.3 (Mukharjee et al., 7]). Let $\mathscr{A}$ and $\mathscr{B}$ be two covers of a topological space $X . \mathscr{A}$ is said to be an s-refinement of $\mathscr{B}$ if for each $A \in \mathscr{A}$ there is a $B \in \mathscr{B}$ such that $A \subsetneq B$. An $s$-refinement $\mathscr{A}$ of $\mathscr{B}$ is said to be an open s-refinement of $\mathscr{B}$ if all members of $\mathscr{A}$ are open.

Definition 2.4 (Mukharjee et al., 7]). An open cover $\mathscr{A}$ is said to be a maximal open cover of $X$ if it is not an s-refinement of any other open cover of $X$.

Definition 2.5 (Mukharjee et al., [7). A topological space $X$ is said to be $m$ compact if each maximal open cover of $X$ has an open finite $s$-refinement.

Definition 2.6 (Mukharjee et al., 7]). Let $X$ and $Y$ be topological spaces. A function $f: X \rightarrow Y$ is said to be m-continuous if $f^{-1}(U)$ is a maximal open set in $X$ for each proper open set $U$ in $Y$.

Definition 2.7 (Mukharjee et al., [7]). A point $x$ of a topological space $X$ is said to be $m$-complete accumulation point of a subset $K$ of $X$ if $|G \cap K|=|K|$ for each maximal open set $G$ containing $x$.

Definition 2.8 (Benchalli et al., 2]). A topological space $X$ is said to be minimal $c$-regular if for each $x \in X$ and each minimal closed set $F$ with $x \notin F$, there exist disjoint open sets $U, V$ such that $x \in U$ and $F \subseteq V$.

Definition 2.9 (Benchalli et al., [3]). A topological space $X$ is said to be minimal $c$-normal if for each pair of distinct minimal closed sets $E, F$, there exist disjoint open sets $U, V$ such that $E \subseteq U$ and $F \subseteq V$.

Definition 2.10 (Mukharjee and Bagchi, [6]). An open set $M$ of $X$ is said to be a mean open set $X$ if there exist two proper open sets $U$ and $V$ of $X$ satisfying $U \varsubsetneqq M \varsubsetneqq V$.

Lemma 1 (Mukharjee et al., 7]). An open cover containing a maximal open set is maximal.

Theorem 1 (Willard, [11]). If $X$ is a $T_{3}$ topological space, then following are equivalent:

(i) $X$ is paracompact,

(ii) each open cover of $X$ has an open $\sigma$-locally finite refinement,

(iii) each open cover of $X$ has a locally finite refinement (not necessarily open),

(iv) each open cover of $X$ has a closed locally finite refinement,

Theorem 2 (Nakaoka and Oda, 9]). If $U$ is a maximal open set and $W$ is an open set in $X$, then either $U \cup W=X$ or $W \subseteq U$. If $W$ is also a maximal open set distinct from $U$, then $U \cup W=X$. 
Theorem 3 (Nakaoka and Oda, [10). If $E$ is a minimal closed set and $F$ is a closed set in $X$, then either $E \cap F=\emptyset$ or $E \subseteq F$. If $F$ is also a minimal closed set distinct from $E$, then $E \cap F=\emptyset$.

Theorem 4 (Mukharjee et al., 7]). Every infinite $T_{1}$ connected topological space is m-compact.

\section{Main results}

Definition 3.1. A topological space $X$ is said to be weakly m-compact if each maximal open cover of $X$ has an open finite refinement.

Let $Y \subseteq X . Y$ is said to be a weakly m-compact subset of $X$ if $\left(Y, \mathscr{T}_{Y}\right)$ is weakly $m$-compact.

Theorem 5. Let $X$ be a m-compact topological space and $F$ be minimal closed in $X$. Then $F$ is weakly $m$-compact.

Proof. Let $\mathscr{A}$ be a maximal open cover of the minimal closed set $F$. Then for each $A \in \mathscr{A}$, there is an open set $B_{A}$ in $X$ such that $A=F \cap B_{A}$. Now $X-F$ is a maximal open set in $X$, and so, by the Lemma 1, $\mathscr{B}=\left\{B_{A}: A \in \mathscr{A}\right\} \cup\{X-F\}$ is a maximal open cover of $X$. By $m$-compactness of $X, \mathscr{B}$ has an open finite $s$-refinement $\left\{U_{1}, U_{2}, \ldots, U_{n}\right\}$, say. Clearly $\left\{U_{1} \cap F, U_{2} \cap F, \ldots, U_{n} \cap F\right\}$ is an open finite refinement of $\mathscr{A}$.

Definition 3.2. Let $X$ be a topological space, $A \subseteq X$ and $x \in X . x$ is said to be an $m$-accumulation point of $A$ if for each maximal open set containing $x$ contains at least one point of $A$ other than $x$.

Theorem 6. Let $X$ be an m-compact topological space. Then every infinite subset of $X$ has an m-accumulation point.

Proof. Let $A$ be an infinite subset of $X$. If possible, let $A$ has no $m$-accumulation point. Then for each $x \in X$, there is a maximal open set $U_{x}$ in $X$ such that $x \in U_{x}$ and $U_{x} \cap A=\emptyset$ or $U_{x} \cap A=\{x\}$. Now $\mathscr{A}=\left\{U_{x}: x \in X\right\}$ is a maximal open cover of $X$ (by the Lemma 1). By the $m$-compactness of $X$, there is a finite $s$-refinement $\mathscr{B}$ of $\mathscr{A}$. Let us write $\mathscr{B}=\left\{B_{x_{1}}, B_{x_{2}}, \ldots, B_{x_{n}}\right\}$. Then $A \subseteq X=\bigcup_{i=1}^{n} B_{x_{i}}$. But for each $i \in\{1,2, \ldots, n\}, A \cap B_{x_{i}}=\emptyset$ or $A \cap B_{x_{i}}=\left\{x_{i}\right\}$. This means that cardinality of $A$ is at most $n$. Which contradicts the fact that $A$ is infinite.

Definition 3.3. A topological space $X$ is said to be $m$-Lindelöf if every maximal open cover of $X$ has an open countable $s$-refinement.

Theorem 7. Let $X$ and $Y$ be topological spaces, where $X$ is $m$-Lindelöf and $f: X \rightarrow Y$ be a bijective $m$-continuous function. Then $Y$ is also $m$-Lindelöf.

Proof. Let $\mathscr{A}^{(Y)}$ be a maximal open cover of $Y$. Since $f$ is a bijective $m$-continuous function, $\mathscr{A}^{(X)}=\left\{f^{-1}(A): A \in \mathscr{A}^{(Y)}\right\}$ is a maximal open cover of $X$ (by Definition 2.6 and Lemma 1). By $m$-Lindelöfness of $X, \mathscr{A}^{(X)}$ has an open countable $s$-refinement $\mathscr{A}_{1}^{(X)}=\left\{B_{\lambda}: \lambda \in \Lambda\right\}$, say, where the index set $\Lambda$ is countable. Since $f$ is bijective, it follows that $\mathscr{A}_{1}^{(Y)}=\left\{f\left(B_{\lambda}\right): \lambda \in \Lambda\right\}$ covers $Y$. Now, let $f\left(B_{\lambda}\right)$ 
be a member of $\mathscr{A}_{1}^{(Y)}$. Then $B_{\lambda} \in \mathscr{A}_{1}^{(X)}$. Since $\mathscr{A}_{1}^{(X)}$ is an $s$-refinement of $\mathscr{A}^{(X)}$, we have $B_{\lambda} \varsubsetneqq f^{-1}(A)$, for some $A \in \mathscr{A}^{(Y)}$. Again since $f$ is bijective, it follows that $f\left(B_{\lambda}\right) \varsubsetneqq A$. Therefore $\mathscr{A}_{1}^{(Y)}$ is an open countable $s$-refinement of $\mathscr{A}^{(Y)}$.

Theorem 8. Let $X$ be an $m$-Lindelöf topological space and $K$ be a subset of $X$ with $|K| \geq$ c. Then $K$ has a complete $m$-accumulation point.

Proof. Suppose for each $x \in X$, there is a maximal open set $V_{x}$ containing $x$ and satisfying $\left|V_{x} \cap K\right|<|K|$. Then $\left|V_{x} \cap K\right| \leq \aleph_{0}$, for each $x \in X$. Since $\left\{V_{x}: x \in X\right\}$ is an open cover of $X$ consists of maximal open sets, by Lemma 1. $\left\{V_{x}: x \in X\right\}$ is a maximal open cover of $X$. So there is an open countable $s$-refinement $\left\{V_{x_{i}}: x_{i} \in X, i \in \Lambda\right\}$, where the index set $\Lambda$ is a countable subset, of $\left\{V_{x}: x \in X\right\}$. Now $|K|=\bigcup_{i \in \Lambda}\left(V_{x_{i}} \cap K\right) \leq \aleph_{0}$. But this implies that $|K| \leq \aleph_{0}<\mathrm{c} \leq|K|$, which is a contradiction.

Theorem 9. Let $X$ be an $m$-Lindelöf topological space and $K$ be an uncountable subset of $X$. Then $K$ has a $m$-accumulation point.

Proof. If possible, let $K$ has no $m$-accumulation point. Then for each $x \in X$, there is a maximal open set $U_{x}$ in $X$ such that $x \in U_{x}$ and $U_{x} \cap K=\emptyset$ or $U_{x} \cap K=\{x\}$. Now $\mathscr{A}=\left\{U_{x}: x \in X\right\}$ is a maximal open cover of $X$ (by the Lemma 1). By the $m$-Lindelöfness of $X$, there is an open countable $s$-refinement $\mathscr{B}$ of $\mathscr{A}$. Let us write $\mathscr{B}=\left\{B_{x_{1}}, B_{x_{2}}, \ldots, B_{x_{n}}, \ldots\right\}$. Then $K \subseteq X=\bigcup_{i=1}^{\infty} B_{x_{i}}$. But for each $i \in\{1,2, \ldots, n, \ldots\}, K \cap B_{x_{i}}=\emptyset$ or $K \cap B_{x_{i}}=\left\{x_{i}\right\}$. This means that cardinality of $K$ is at most $\aleph_{0}$. Which contradicts the fact that $K$ is uncountable.

Definition 3.4. A topological space $X$ is said to be weakly $m$-Lindelöf if each maximal open cover of $X$ has a countable open refinement.

Let $Y \subseteq X$. $Y$ is said to be a weakly $m$-compact subset of $X$ if $\left(Y, \mathscr{T}_{Y}\right)$ is weakly $m$-compact.

Theorem 10. Let $X$ be a m-Lindelöf topological space and $F$ be minimal closed in $X$. Then $F$ is weakly $m$-Lindelöf.

Proof. Proof is similar to the proof of Theorem 5

Definition 3.5. A topological space $X$ is said to be countably m-compact if every countable maximal open cover has a finite open $s$-refinement.

Obviously an $m$-compact topological space is countably $m$-compact.

Theorem 11. Let $X$ be a Lindelöf topological space containing a minimal closed set $F$. Then following are equivalent:

(i) $X$ is m-compact.

(ii) $X$ is countably $m$-compact.

Proof. $(i) \Rightarrow(i i)$

This part is obvious.

(ii) $\Rightarrow($ i)

Let $\mathscr{A}$ be a maximal open cover of $X$. By Lindelöfness of $X, \mathscr{A}$ has a countable subcollection $\mathscr{B}$, say, that covers $X$. Then by the Lemma 1 , $\mathscr{B} \cup\{X-F\}$ is 
a countable maximal open cover of $X$. By the countably $m$-compactness of $X$, $\mathscr{B} \cup\{X-F\}$ has a finite open $s$-refinement of $X$, i.e., $X$ is $m$-compact.

Theorem 12. An infinite $T_{1}$ connected topological space is countably $m$-compact.

Proof. Proof follows from Theorem 4.

Definition 3.6. A topological space $X$ is said to be an $m$-paracompact topological space if each maximal open cover of $X$ has an open locally finite $s$-refinement.

Theorem 13. If $X$ is an m-paracompact topological space, then each maximal open cover of $X$ has an open $\sigma$-locally finite s-refinement.

Proof. Proof is trivial.

Lemma 2. Let $\mathscr{A}$ be an s-refinement (resp., refinement) of $\mathscr{B}$ and $\mathscr{B}$ be a refinement (resp., s-refinement) of $\mathscr{C}$. Then $\mathscr{A}$ is an s-refinement of $\mathscr{C}$.

Proof. Proof is very easy and hence omitted.

Theorem 14. If $X$ is a m-paracompact topological space, then each maximal open cover of $X$ has a locally finite s-refinement (not necessarily open).

Proof. Proof follows from the Lemma 2 and the Theorem 1.

Theorem 15. A Hausdorff m-paracompact topological space is minimal c-regular.

Proof. Let $X$ be a Hausdorff $m$-paracompact topological space. Also let $x \in X$ and $F$ be a minimal closed set such that $x \notin F$. Then for each $y \in F$, there exist disjoint open sets $U_{y}, V_{y}$ such that $x \in U_{y}$ and $y \in V_{y}$. Clearly $x \notin \operatorname{cl}\left(V_{y}\right)$. Then $\mathscr{V}=\left\{V_{y}: y \in F\right\} \cup\{X-F\}$ is a maximal open cover of $X$, by the Lemma 1 Since $X$ is $m$-paracompact, there is an open locally finite $s$-refinement $\mathscr{W}$, say, of $\mathscr{V}=\left\{V_{y}: y \in F\right\} \cup\{X-F\}$.

Let $V=\bigcup\{W \in \mathscr{W} \mid W \cap F \neq \emptyset\}$. Then $V$ is an open set which contains $F$. Since $\{W \in \mathscr{W} \mid W \cap F \neq \emptyset\}$ is a subcollection of a locally finite family, it is locally finite and therefore $\operatorname{cl}(V)=\bigcup\{c l(W): W \in \mathscr{W}, W \cap F \neq \emptyset\}$. Now for each $W \in \mathscr{W}$, there is a $V_{y} \in \mathscr{V}$ such that $W \subsetneq V_{y}$, i.e., $\operatorname{cl}(W) \subseteq \operatorname{cl}\left(V_{y}\right)$. Thus $x \notin c l(V)$, i.e., $x \in X-\operatorname{cl}(V)$. Thus $X$ is minimal $c$-regular.

Corollary 15.1. A Hausdorff m-paracompact topological space is minimal cnormal.

Proof. Let $E$ and $F$ distinct minimal closed sets in a Hausdorff $m$-paracompact topological space $X$. For each $y \in E$, by the Theorem 15 there exist disjoint open sets $U_{y}, V_{y}$ such that $y \in U_{y}$ and $B \subseteq V_{y}$. Again by the Lemma 1 ,

$$
\mathscr{U}=\left\{U_{y}: y \in E\right\} \cup\{X-E\}
$$

is a maximal open cover of $X$. Now if we proceed in a similar way as the proof of the Theorem, we can get disjoint open sets $U, V$ such that $E \subseteq U$ and $F \subseteq V$. 
Definition 3.7. A topological space $X$ is said to be weakly m-paracompact if each maximal open cover of $X$ has an open locally finite refinement.

Let $Y \subseteq X . Y$ is said to be a weakly m-paracompact subset of $X$ if $\left(Y, \mathscr{T}_{Y}\right)$ is weakly $m$-paracompact.

Theorem 16. Let $X$ be an m-paracompact topological space and $F$ be minimal closed in $X$. Then $F$ is weakly m-paracompact.

Proof. Proof is similar to the proof of Theorem 5 .

Theorem 17. Let $X$ be a topological space in which all proper open sets are mean open. Then $X$ is m-compact.

Proof. If possible, let $X$ be not $m$-compact. Then there exists a maximal open cover $\mathscr{A}$ of $X$ which has no open finite $s$-refinement. Now by the Definition 2.10 , for each $A \in \mathscr{A}$ we have can a proper open set $B_{A}$ such that $A \subsetneq B_{A}$. Then $\mathscr{A}$ is an $s$-refinement of $\left\{B_{A}: A \in \mathscr{A}\right\}$, which contradicts the maximality of $\mathscr{A}$. Thus $X$ is $m$-compact.

Corollary 17.1. Let $X$ be a topological space in which all proper open sets are mean open. Then $X$ is $m$-Lindelöf, countably $m$-compact, m-paracompact, weakly $m$-compact, weakly $m$-Lindelöf and weakly m-paracompact.

Proof. Proof is similar to the proof of the previous theorem.

\section{ACKNOWLEDGMENT}

The author is grateful to the referee for examining the paper carefully and for suggesting some necessary corrections in the paper.

\section{REFERENCES}

[1] K. B. Bagchi and A. Mukharjee, On maximal, minimal and mean open sets, Afr. mat., 30 (1-2), (2019), 291-296.

[2] S. S. Benchalli, B. M. Ittanagi and R. S. Wali, On minimal separation axioms in topological spaces, J. Adv. Stud. Topol. 3 (2012), 98-104.

[3] S. S. Benchalli, B. M. Ittanagi and R. S. Wali, On minimal normal and minimal compact spaces, J. Comput. Math. Sci. 3 (2012), 280-286.

[4] A. Mukharjee, On maximal, minimal open and closed sets, Commun. Korean Math. Soc., $30(3),(2015), 277-282$.

[5] A. Mukharjee, More on maximal, minimal open and closed sets, Commun. Korean Math. Soc., 3(1), (2017), 175-181.

[6] A. Mukharjee and K. B. Bagchi, On mean open and closed sets, Kyungpook Math. J., 56(4), (2016), 1259-1265.

[7] A. Mukharjee, S. Raut and K. B. Bagchi, Compactness and regularity via maximal open and minimal closed sets, Sci. Stud. Res. Ser. Math. Inform., 28(1), (2018), 53-60.

[8] F. Nakaoka, N. Oda, Some applications of minimal open sets, Int. J. Math. Math. Sci., 27(8), (2001), 471-476

[9] F. Nakaoka, N. Oda, Some properties of maximal open sets, Int. J. Math. Math. Sci., 2003(21), (2003), 1331-1340 
[10] F. Nakaoka, N. Oda, Minimal closed sets and maximal, Int. J. Math. Math. Sci., 2006, Article ID 18647, 8 pages, doi:10.1155/IJMMS/2006/18647.

[11] S. Willard, General topology, Addison-wesley publishing company, 1970.

1 Kalipada Ghosh Tarai Mahavidyalaya,

Department of Mathematics,

Siliguri, W. Bengal-734014, India

Email address: kbagchi.789@gmail.com

Received 19.5.2021

Revised 28.8.2021

Accepted 30.8.2021 